\title{
Association of nonsteroidal anti-inflammatory drugs and aspirin use and the risk of head and neck cancers: a meta-analysis of observational studies
}

\author{
Lanhua Tang ${ }^{1, *}$, Huabin Hu${ }^{2, *}$, Huai Liu ${ }^{3,4}$, Chengzhu Jian ${ }^{5}$, Hui Wang ${ }^{3,4}$, Jin Huang ${ }^{1}$ \\ ${ }^{1}$ Department of Oncology, Xiangya Hospital of Central South University, Changsha, China \\ ${ }^{2}$ Department of Medical Oncology, The Sixth Affiliated Hospital of Sun Yat-Sen University, Guangzhou, China \\ ${ }^{3}$ Department of Radiotherapy, Hunan Cancer Hospital and The Affiliated Cancer Hospital of Xiangya School of Medicine, \\ Central South University, Changsha, China \\ ${ }^{4}$ Key Laboratory of Translational Radiation Oncology, Changsha, China \\ ${ }^{5}$ Department of Urology, The Second Xiangya Hospital of Central South University, Changsha, China \\ * Lanhua Tang and Huabin Hu contributed equally to this work \\ Correspondence to: Jin Huang, email: jinhuang@csu.edu.cn \\ Keywords: head and neck cancer, nonsteroidal anti-inflammatory drugs, aspirin, chemoprevention, meta-analysis \\ Received: May 02, $2016 \quad$ Accepted: July 28, $2016 \quad$ Published: August 12, 2016
}

\section{ABSTRACT}

Purpose: Nonsteroidal anti-inflammatory drugs (NSAIDs), including aspirin, have emerged as the potential chemopreventive agents for a number of cancer types, however, previous studies of head and neck cancers (HNC) have yielded inconclusive results. We performed a meta-analysis of observational studies to quantitatively assess the association between NSAIDs use and the risk for HNC.

Methods: We searched Pubmed, Embase, Google scholar, and Cochrane library for relevant studies that were published in any language, from January 1980 to April 2016. We pooled the odds ratio (OR) from individual studies and performed subgroup, heterogeneity, and publication bias analyses.

Results: A total of eleven studies (eight case-control studies and three cohort studies), involving 370,000 participants and 10,673 HNC cases contributed to this meta-analysis. The results of these studies suggested that neither use of overall NSAIDs (OR=0.95; 95\% CI, 0.81-1.11), aspirin (OR=0.93; 95\% CI, 0.79-1.10), nor nonsteroidal NSAIDs (OR=0.92; 95\% CI, 0.76-1.10) were associated with HNC risk. Similar nonsteroidal results were observed when stratified by HNC sites, study design, sample size, and varied adjustment factors. However, we found significant protective effect of ibuprofen ( $\mathrm{OR}=0.85 ; 95 \% \mathrm{CI}, 0.72-0.99)$ and long-term aspirin use ( $\geqq 5$ years) $(\mathrm{OR}=0.75 ; 95 \% \mathrm{CI}, 0.65-0.85)$ on $\mathrm{HNC}$ risk, with low heterogeneity and publication bias.

Conclusions: Our meta-analysis results do not support the hypothesis that overall use of NSAIDs significant reduces the risk of HNC. Whereas, we cannot rule out a modest reduction in HNC risk associated with ibuprofen and long-term aspirin use.

\section{INTRODUCTION}

Head and neck cancers (HNC) continues to be an important public health problem, with an estimated 700,000 new cases around the world in 2012 [1]. HNC includes a variety of cancers originating from different sites within the head and neck region, such as the oral cavity, oropharynx, hypopharynx and larynx. It is well documented that tobacco consumption and excessive alcohol drinking are independent major risk factors for the development of HNC [2-4]. Other possible risk factors include infection with human papilloma virus (HPV), poor oral hygiene, environmental carcinogens and genetic susceptibility [5-9]. Despite great advance in multidisciplinary treatment, approximately 30 $50 \%$ patients with HNC survive over 5 years after initial diagnosis, and $15 \%-20 \%$ patients will develop second primary malignancies after extensive therapy 
$[10,11]$. Therefore, it is crucial that identifying potential chemopreventive measures other than tobacco and alcohol cessation should be further investigated.

Convincing laboratory evidence has emerged to demonstrate an association between chronic inflammation and cancer, which makes the anti-inflammatory drugs have emerged as the most potential chemopreventive agents [12-15]. Nonsteroidal anti-inflammatory drugs (NSAIDs), have received increasing attention because of their inhibitive effect on the cyclo-oxygenase (COX) enzymes, which may prevent synthesis of prostaglandins that stimulate growth and play a role in promoting carcinogenesis [16-19].

Several epidemiologic studies have observed use of aspirin and other NSAIDs have an association with reduced risk for cancers of the colon, stomach, prostate and breast [17, 20-22]. Even though some studies supported NSAIDs use significantly decrease the risk of HNC [11, 23, 24], while other studies did not show a consistent benefit $[25,26]$. On the basis of the previous systematic review by Wilson et al. in 2011 [27], no definitive conclusion can be reached on NSAID/aspirin use and $\mathrm{HNC}$ risk. In addition, no meta-analysis and quantitative analysis was conducted due to the limited case number and heterogeneity of the studies identified. The purpose of the present study was to summarize all available evidence on this issue using a meta-analysis of observational studies.

\section{RESULTS}

\section{Eligible studies}

The overview of our search process was illustrated in Figure 1. Eleven studies met the predetermined criteria

$$
\begin{aligned}
& \text { Potentially relevant articles identified } \\
& \text { from search of PubMed ( } n=2301) \text {, } \\
& \text { Embase ( } n=703) \text {, Google scholar } \\
& (n=2050) \text { and Cochrane library }(n=147)
\end{aligned}
$$

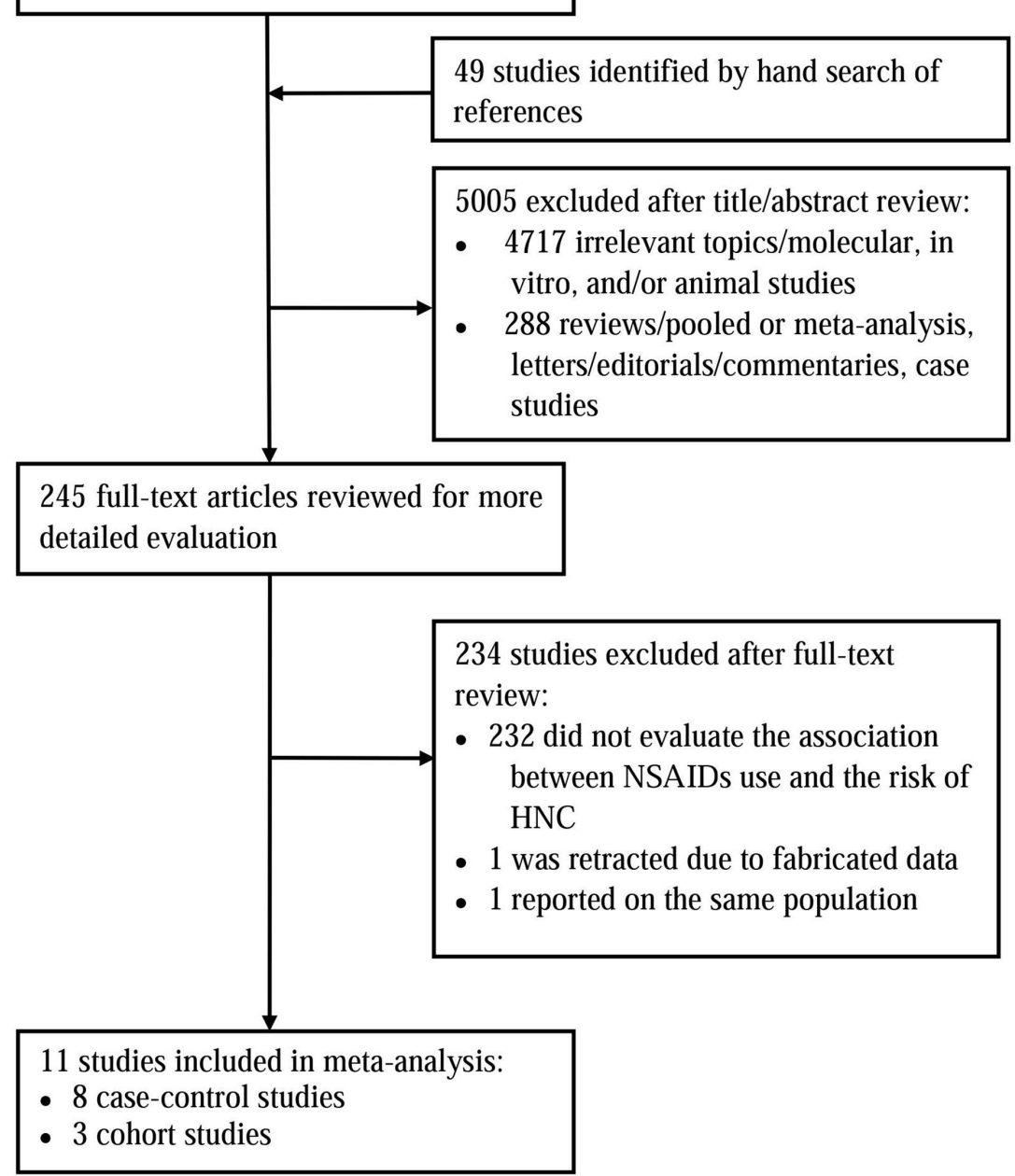

Figure 1: The flow diagram of search strategy. 
for inclusion, with three cohort studies [24, 28, 29], and eight case-control studies [11, 23, 25, 26, 30-33]. The number of HNC cases ranged from 71 to 2,745 in the casecontrol studies, and from 68 to 185 in the cohort studies. Three studies were conducted in the United States [11, 24, 31], two in Denmark [28, 29], two in United Kingdom $[26,32]$, two in Italy [23, 33], one in Sweden [30] and the other one was coordinated by the International Agency for Research on Cancer (IARC) in fourteen European centers (in ten countries) [25]. These eleven studies were published between 2003 and 2015. The range of enrollment periods for participants across studies was 1982-2013. Table 1 and Supplementary Table S1 listed the study characteristics and corresponding estimated OR with $95 \%$ CIs.

\section{Quality of study methodologies}

Table 2 showed the quality of study methodology included in the meta-analysis. The range of quality scores was 5 - 8; the average score was 6.7. The average scores of cohort studies and case-control studies were 7.7 and 6.4, respectively, which suggests a reasonable good quality of the cohort and case-control studies.

\section{Overall use of NSAIDs and the risk for HNC}

Figure 2 illustrated the forest plot of ORs estimates with $95 \%$ CIs from individual studies and overall meta-analysis of all eleven studies [11, 23-26, 28-33]. The overall summary ORs demonstrated no significant association between overall

Table 1: Characteristics of studies included in the meta-analysis of NSAIDs use and the risk of HNC

\begin{tabular}{|c|c|c|c|c|c|c|c|c|c|}
\hline Study & $\begin{array}{l}\text { Study } \\
\text { design }\end{array}$ & $\begin{array}{c}\text { Study } \\
\text { location }\end{array}$ & Period & HNC sites & Cases & $\begin{array}{l}\text { Controls } \\
\text { or cohort } \\
\text { size }\end{array}$ & $\begin{array}{l}\text { Type of } \\
\text { drugs }\end{array}$ & OR $(95 \% C I)$ & $\begin{array}{l}\text { Adjustment } \\
\text { for covariates }\end{array}$ \\
\hline \multirow[t]{2}{*}{$\begin{array}{l}\text { Bosetti et al, } \\
2003\end{array}$} & \multirow[t]{2}{*}{$\begin{array}{l}\text { Case- } \\
\text { control }\end{array}$} & \multirow[t]{2}{*}{ Italy } & \multirow[t]{2}{*}{$1992-2000$} & \multirow[t]{2}{*}{$\begin{array}{l}\text { Combined sites } \\
\text { (oral, pharynx } \\
\text { and larynx) }\end{array}$} & 965 & 1779 & $\begin{array}{c}\text { Aspirin (at } \\
\text { least once a } \\
\text { week for more } \\
\text { than } 6 \text { months) }\end{array}$ & $0.86(0.46-1.61)^{\mathrm{a}}$ & \multirow{2}{*}{$\begin{array}{l}\text { Age, gender, } \\
\text { centre, } \\
\text { educational, } \\
\text { smoking, } \\
\text { alcohol } \\
\text { drinking }\end{array}$} \\
\hline & & & & & 935 & 1732 & $\begin{array}{l}\text { Long-term } \\
\text { aspirin use } \\
\text { (duration of } \\
\text { use } \geqq 5 \text { years) }\end{array}$ & $0.33(0.13-0.82)$ & \\
\hline \multirow{3}{*}{$\begin{array}{l}\text { Friis } \\
\text { et al, } 2003\end{array}$} & \multirow[t]{3}{*}{ Cohort } & \multirow[t]{3}{*}{ Denmark } & \multirow[t]{3}{*}{$1989-1995$} & Combined sites & 68 & \multirow[t]{3}{*}{29470} & \multirow{3}{*}{$\begin{array}{c}\text { Aspirin }(75, \\
100 \text { or } 150 \\
\mathrm{mg})\end{array}$} & $1.36(1.04-1.77)^{\mathrm{b}}$ & \multirow[t]{3}{*}{ Age, gender } \\
\hline & & & & $\begin{array}{l}\text { Oral and } \\
\text { oropharynx }\end{array}$ & 48 & & & $1.30(0.90-1.70)$ & \\
\hline & & & & Larynx & 20 & & & $1.50(0.90-2.40)$ & \\
\hline $\begin{array}{l}\text { Rosenquist et al, } \\
2005\end{array}$ & $\begin{array}{l}\text { Case- } \\
\text { control }\end{array}$ & Sweden & $2000-2004$ & $\begin{array}{c}\text { Oral and } \\
\text { oropharynx }\end{array}$ & 132 & 320 & $\begin{array}{c}\text { Aspirin } \\
\text { (75-150 mg/ } \\
\text { day) }\end{array}$ & $1.00(0.60-1.70)^{\mathrm{a}}$ & $\begin{array}{l}\text { Smoking, } \\
\text { alcohol } \\
\text { drinking }\end{array}$ \\
\hline $\begin{array}{l}\text { Friis } \\
\text { et al, } 2006\end{array}$ & Cohort & Denmark & $1991-2002$ & $\begin{array}{c}\text { Oral (month and } \\
\text { tongue) }\end{array}$ & 185 & 169589 & $\begin{array}{l}\text { NA-NSAIDs } \\
\quad(\geqq 2 \\
\text { prescriptions })\end{array}$ & $1.20(1.00-1.60)$ & Age, gender \\
\hline \multirow{7}{*}{$\begin{array}{l}\text { Jayaprakash } \\
\text { et al, } 2006\end{array}$} & \multirow{7}{*}{$\begin{array}{l}\text { Case- } \\
\text { control }\end{array}$} & \multirow{7}{*}{$\begin{array}{l}\text { United } \\
\text { States }\end{array}$} & \multirow[t]{7}{*}{$1982-1998$} & Combined sites & 529 & 529 & Aspirin & $0.75(0.58-0.96)$ & \multirow{7}{*}{$\begin{array}{l}\text { Age, gender, } \\
\text { smoking, } \\
\text { alcohol } \\
\text { drinking }\end{array}$} \\
\hline & & & & Oral cavity & 169 & 169 & (ever used) & $0.73(0.51-1.05)$ & \\
\hline & & & & Nasopharynx & 22 & 22 & & $0.88(0.36-2.15)$ & \\
\hline & & & & Oropharynx & 102 & 102 & & $0.68(0.44-1.05)$ & \\
\hline & & & & Larynx & 141 & 141 & & $0.89(0.58-1.35)$ & \\
\hline & & & & Hypopharynx & 31 & 31 & & $0.77(0.35-1.66)$ & \\
\hline & & & & Combined sites & 313 & 289 & $\begin{array}{l}\text { Long-term } \\
\text { aspirin use } \\
\text { (duration } \\
\text { of use } \geqq 10 \\
\text { years) }\end{array}$ & $0.65(0.52-0.82)^{\mathrm{c}}$ & \\
\hline \multirow[t]{3}{*}{$\begin{array}{l}\text { Ahmadi } \\
\text { et al, } 2010\end{array}$} & \multirow[t]{3}{*}{$\begin{array}{l}\text { Case- } \\
\text { control }\end{array}$} & \multirow[t]{3}{*}{$\begin{array}{l}\text { United } \\
\text { States }\end{array}$} & \multirow[t]{3}{*}{$2003-2007$} & \multirow[t]{3}{*}{ Combined sites } & 71 & 71 & $\begin{array}{l}\text { Any NSAIDs } \\
\text { (ever used) }\end{array}$ & $0.31(0.11-0.88)$ & \multirow[t]{3}{*}{$\begin{array}{l}\text { Educational, } \\
\text { marital status }\end{array}$} \\
\hline & & & & & 44 & 47 & $\begin{array}{l}\text { Any NSAIDs } \\
\text { (daily) }\end{array}$ & $0.14(0.04-0.54)$ & \\
\hline & & & & & 25 & 25 & Aspirin (daily) & $0.15(0.02-1.30)$ & \\
\hline
\end{tabular}

(Continued) 


\begin{tabular}{|c|c|c|c|c|c|c|c|c|c|}
\hline Study & $\begin{array}{l}\text { Study } \\
\text { design }\end{array}$ & $\begin{array}{c}\text { Study } \\
\text { location }\end{array}$ & Period & HNC sites & Cases & $\begin{array}{l}\text { Controls } \\
\text { or cohort } \\
\text { size }\end{array}$ & $\begin{array}{l}\text { Type of } \\
\text { drugs }\end{array}$ & OR $(95 \% \mathrm{CI})$ & $\begin{array}{c}\text { Adjustment } \\
\text { for covariates }\end{array}$ \\
\hline \multirow{7}{*}{$\begin{array}{l}\text { Macfarlane et } \\
\text { al, } 2012\end{array}$} & \multirow{7}{*}{$\begin{array}{l}\text { Case- } \\
\text { control }\end{array}$} & \multirow[t]{7}{*}{ European } & \multirow[t]{7}{*}{ NR } & Combined sites & 1779 & 1993 & \multirow{6}{*}{$\begin{array}{c}\text { Aspirin (at } \\
\text { least once a } \\
\text { week for one } \\
\text { year) }\end{array}$} & $0.92(0.78-1.09)^{b}$ & \multirow{7}{*}{$\begin{array}{l}\text { Age, gender, } \\
\text { BMI, } \\
\text { educational, } \\
\text { smoking, } \\
\text { alcohol } \\
\text { drinking, fruit } \\
\text { consumption }\end{array}$} \\
\hline & & & & Oral cavity & 510 & 1993 & & $1.04(0.76-1.41)$ & \\
\hline & & & & Oropharynx & 474 & 1993 & & $1.05(0.76-1.46)$ & \\
\hline & & & & OP NOS & 112 & 1993 & & $1.29(0.70-2.35)$ & \\
\hline & & & & Larynx & 670 & 1993 & & $0.74(0.54-1.01)$ & \\
\hline & & & & Hypopharynx & 183 & 1993 & & $0.53(0.28-1.02)$ & \\
\hline & & & & Combined sites & 1588 & 1736 & $\begin{array}{l}\text { Long-term } \\
\text { aspirin use } \\
\text { (duration } \\
\text { of use } \geqq 5 \\
\text { years) }\end{array}$ & $0.78(0.58-1.05)^{d}$ & \\
\hline \multirow[t]{3}{*}{$\begin{array}{l}\text { Wilson et al, } \\
2013\end{array}$} & \multirow[t]{3}{*}{ Cohort } & \multirow[t]{3}{*}{$\begin{array}{l}\text { United } \\
\text { States }\end{array}$} & \multirow[t]{3}{*}{$1993-2001$} & \multirow[t]{3}{*}{ Combined sites } & 316 & 141718 & $\begin{array}{c}\text { Aspirin } \\
\text { (regular use) }\end{array}$ & $0.78(0.62-0.98)$ & \multirow[t]{2}{*}{$\begin{array}{l}\text { Age, gender, } \\
\text { BMI, smoking }\end{array}$} \\
\hline & & & & & 315 & 141550 & $\begin{array}{c}\text { Aspirin } \\
\text { (daily) }\end{array}$ & $0.85(0.65-1.11)$ & \\
\hline & & & & & 316 & 141718 & $\begin{array}{l}\text { Ibuprofen } \\
\text { (regular use) }\end{array}$ & $0.97(0.75-1.27)$ & $\begin{array}{l}\text { Age, gender, } \\
\text { BMI, } \\
\text { smoking, } \\
\text { aspirin use }\end{array}$ \\
\hline \multirow[t]{3}{*}{$\begin{array}{l}\text { Macfarlane et } \\
\text { al, } 2014\end{array}$} & \multirow[t]{3}{*}{$\begin{array}{l}\text { Case- } \\
\text { control }\end{array}$} & \multirow[t]{3}{*}{$\begin{array}{l}\text { United } \\
\text { Kingdom }\end{array}$} & \multirow[t]{3}{*}{$1996-2010$} & \multirow[t]{3}{*}{ Combined sites } & 2392 & 7165 & $\begin{array}{c}\text { Aspirin }(\geqq 1 \\
\text { prescriptions) }\end{array}$ & $0.93(0.76-1.15)$ & \multirow{3}{*}{$\begin{array}{l}\text { Age, gender, } \\
\text { carstairs } \\
\text { deprivation } \\
\text { category, } \\
\text { coronary heart } \\
\text { disease, stroke }\end{array}$} \\
\hline & & & & & 2034 & 6094 & $\begin{array}{l}\text { Long-term } \\
\text { aspirin } \\
\text { use (time } \\
\text { between } \\
\text { last and first } \\
\text { prescription } \\
\geqq 5.4 \text { years) }\end{array}$ & $0.85(0.70-1.04)$ & \\
\hline & & & & & 2392 & 7165 & $\begin{array}{c}\text { NA-NSAIDs } \\
(\geqq 1 \\
\text { prescriptions })\end{array}$ & $0.82(0.70-0.96)$ & \\
\hline \multirow[t]{2}{*}{$\begin{array}{l}\text { Becker et al, } \\
2015\end{array}$} & \multirow[t]{2}{*}{$\begin{array}{l}\text { Case- } \\
\text { control }\end{array}$} & \multirow[t]{2}{*}{$\begin{array}{c}\text { United } \\
\text { Kingdom }\end{array}$} & \multirow[t]{2}{*}{$1995-2013$} & \multirow[t]{2}{*}{ Combined sites } & 2238 & 13488 & $\begin{array}{l}\text { Aspirin }(\geqq 50 \\
\text { prescriptions) }\end{array}$ & $1.21(0.97-1.51)$ & \multirow{2}{*}{$\begin{array}{l}\text { BMI, } \\
\text { smoking, } \\
\text { alcohol } \\
\text { drinking }\end{array}$} \\
\hline & & & & & 1932 & 11657 & $\begin{array}{c}\text { Ibuprofen } \\
(\geqq 6 \\
\text { prescriptions })\end{array}$ & $0.78(0.64-0.96)$ & \\
\hline $\begin{array}{l}\text { Di Maso et al, } \\
2015\end{array}$ & $\begin{array}{l}\text { Case- } \\
\text { control }\end{array}$ & Italy & $1992-2008$ & Nasopharynx & 198 & 592 & $\begin{array}{l}\text { Aspirin (at } \\
\text { least one } \\
\text { aspirin a } \\
\text { week for } \\
\text { more than } 6 \\
\text { months) }\end{array}$ & $0.24(0.07-0.87)$ & $\begin{array}{l}\text { Age, gender, } \\
\text { area of } \\
\text { residence, } \\
\text { smoking, } \\
\text { period of } \\
\text { interview, } \\
\text { years of } \\
\text { education, } \\
\text { occupation }\end{array}$ \\
\hline
\end{tabular}

Abbreviations: BMI, body mass index; CI, confidence interval; F, female; HNC, Head and Neck Cancers; M, male; NR, not reported; NA-NSAIDs, non-aspirin nonsteroidal anti-inflammatory drugs; NSAIDs, nonsteroidal anti-inflammatory drugs; OP NOS, Oral, pharynx not otherwise specified; OR, odds ratio.a, Cited from a systematic review by Wilson et al.; b, Pooled from all cancer sites except for esophagus; c, Pooled from the ORs of 10-20 years, $21-40$ years and $\geqq 40$ years; $d$, Pooled from the ORs of $5-9$ years and $\geqq 10$ years.

NSAIDs use and the risk of $\mathrm{HNC}(\mathrm{OR}=0.95 ; 95 \% \mathrm{CI}, 0.81$ $1.11)$, with statistical heterogeneity among studies $(P=0.001$; $\left.I^{2}=67.4 \%\right)$. The tests for funnel plot asymmetry by Begg's test and Egger's test identified no publication bias (Begg's test, $P=0.276$; Egger's test, $P=0.229$ ).

\section{Subgroup analyses}

Table 3 showed the associations between NSAIDs use and the risk of HNC in subgroup meta-analyses by various factors. The subgroup analyses were conducted 
Table 2: Methodological quality of included studies based on the Newcastle-Ottawa Scale

\begin{tabular}{|c|c|c|c|c|c|c|c|c|c|}
\hline $\begin{array}{l}\text { Cohort } \\
\text { studies } \\
(n=3)\end{array}$ & $\begin{array}{c}\text { Representativeness } \\
\text { of the exposed } \\
\text { cohort }\end{array}$ & $\begin{array}{c}\text { Selection } \\
\text { of the } \\
\text { unexposed } \\
\text { cohort }\end{array}$ & $\begin{array}{c}\text { Ascertainment } \\
\text { of exposure }\end{array}$ & $\begin{array}{c}\text { Outcome } \\
\text { of } \\
\text { interest } \\
\text { not } \\
\text { present } \\
\text { at start } \\
\text { of study }\end{array}$ & $\begin{array}{c}\text { Control } \\
\text { for } \\
\text { Important } \\
\text { factor or } \\
\text { additional } \\
\text { factor }\end{array}$ & $\begin{array}{c}\text { Assessment } \\
\text { of outcome }\end{array}$ & $\begin{array}{l}\text { Follow-up } \\
\text { long enough } \\
\text { for outcomes } \\
\text { to occur }{ }^{a}\end{array}$ & $\begin{array}{l}\text { Adequacy of } \\
\text { follow-up of } \\
\text { cohorts }\end{array}$ & Total (0-9) \\
\hline $\begin{array}{l}\text { Friis et al, } \\
2003\end{array}$ & $\star$ & $\star$ & $\star$ & $\star$ & $\star$ & $\star$ & $\star$ & $\star$ & 8 \\
\hline $\begin{array}{l}\text { Friis et al, } \\
2006\end{array}$ & $\star$ & $\star$ & $\star$ & $\star$ & $\star$ & $\star$ & $\star$ & $\star$ & 8 \\
\hline $\begin{array}{l}\text { Wilson et al, } \\
2013\end{array}$ & $\star$ & $\star$ & - & $\star$ & $\star$ & $\star$ & $\star$ & $\star$ & 7 \\
\hline $\begin{array}{l}\text { Case-control } \\
\text { studies }(n=7)\end{array}$ & $\begin{array}{l}\text { Adequate definition } \\
\text { of cases }\end{array}$ & $\begin{array}{l}\text { Representa- } \\
\text { tiveness of } \\
\text { cases }\end{array}$ & $\begin{array}{l}\text { Selection of } \\
\text { contros }\end{array}$ & $\begin{array}{c}\text { Definition } \\
\text { of } \\
\text { controls }\end{array}$ & $\begin{array}{l}\text { Control } \\
\text { for } \\
\text { important } \\
\text { factor or } \\
\text { additional } \\
\text { factor }\end{array}$ & $\begin{array}{c}\text { Ascertainment } \\
\text { of exposure }\end{array}$ & $\begin{array}{c}\text { Same } \\
\text { method of } \\
\text { ascertainment } \\
\text { for subjects }\end{array}$ & $\begin{array}{c}\text { Nonresponse } \\
\text { Rate }^{b}\end{array}$ & Total (0-9) \\
\hline $\begin{array}{l}\text { Bosetti et al, } \\
2003\end{array}$ & - & $\star$ & $\star$ & $\star$ & $\star \star$ & - & $\star$ & - & 6 \\
\hline $\begin{array}{l}\text { Rosenquist et } \\
\text { al, } 2005\end{array}$ & $\star$ & $\star$ & $\star$ & $\star$ & $\star$ & - & $\star$ & - & 6 \\
\hline $\begin{array}{l}\text { Jayaprakash } \\
\text { et al, } 2006\end{array}$ & $\star$ & $\star$ & $\star$ & $\star$ & $\star \star$ & - & $\star$ & - & 7 \\
\hline $\begin{array}{l}\text { Ahmadi et al, } \\
2010\end{array}$ & $\star$ & $\star$ & $\star$ & $\star$ & - & - & $\star$ & - & 5 \\
\hline $\begin{array}{l}\text { Macfarlane } \\
\text { et al, } 2012\end{array}$ & $\star$ & $\star$ & $\star$ & $\star$ & $\star \star$ & - & $\star$ & - & 7 \\
\hline $\begin{array}{l}\text { Macfarlane } \\
\text { et al, } 2014\end{array}$ & $\star$ & $\star$ & $\star$ & $\star$ & $\star$ & $\star$ & $\star$ & - & 7 \\
\hline $\begin{array}{l}\text { Becker et al, } \\
2015\end{array}$ & $\star$ & $\star$ & $\star$ & $\star$ & $\star$ & $\star$ & $\star$ & - & 7 \\
\hline $\begin{array}{l}\text { Di Maso et } \\
\text { al, } 2015\end{array}$ & $\star$ & $\star$ & - & $\star$ & $\star$ & - & $\star$ & $\star$ & 6 \\
\hline
\end{tabular}

a, A cohort study with a follow-up time $>5$ years was assigned one star; $b$, Same rate for both groups was assigned one star.

on the basis of different types of NSAIDs use. There was no significant association between use of aspirin [11, 23-26, 28, 30-33] or non-aspirin nonsteroidal antiinflammatory drugs (NA-NSAIDs) [24, 26, 29, 32] and the risk of $\mathrm{HNC}$, with $\mathrm{OR}$ of $0.93(95 \% \mathrm{CI}, 0.79-1.10)$ and OR of 0.92 (95\%CI, 0.76-1.10), respectively. For the two studies evaluated exposure to ibuprofen and the risk of HNC [24, 32], a significant protective effect was observed $(\mathrm{OR}=0.85 ; 95 \% \mathrm{CI}, 0.72-0.99)$. We also found long- term usage of aspirin ( $\geqq 5$ years) has been associated with a significant $25 \%$ reduction in HNC risk from four studies $[11,23,25,26](\mathrm{OR}=0.75 ; 95 \% \mathrm{CI}, 0.65-0.85)$ (Figure 3), and statistical heterogeneity was not detected.

There are six studies provided results on the effect of NSAIDs for specific HNC sites. However, NSAIDs use was not associated with a reduced risk for cancer of oral and oropharynx $[11,25,28-30](\mathrm{OR}=1.01 ; 95 \% \mathrm{CI}, 0.85$ $1.20)$, larynx $[11,25,28](\mathrm{OR}=0.96 ; 95 \% \mathrm{CI}, 0.65-1.42)$, hypopharynx $[11,25](\mathrm{OR}=0.62 ; 95 \% \mathrm{CI}, 0.38-1.01)$ and nasopharynx $[11,33](\mathrm{OR}=0.50 ; 95 \% \mathrm{CI}, 0.14-1.76)$.

To examine consistency across varying study designs with different potential biases, we stratified data into subgroups on the basis of study design. The summary ORs were 0.89 (95\%CI: 0.74 - 1.07) pooled from eight casecontrol studies [11, 23, 25, 26, 30-33] and 1.08 (95\%CI: $0.77-1.51$ ) across three cohort studies [24, 28, 29].

The impact of sample size on risk estimates was assessed. The summary ORs were 0.99 (95\%CI: 0.85 - 1.15) in eight studies [11, 23-26, 28-32] with relatively large sample size $(\geqq 1,000)$ and 0.47 (95\% CI: 0.18 - 1.27) from the other three studies with the sample size less than $1,000[30,31,33]$.

Recorded prescription database, self-administered questionnaires and standardized interviews were used to obtain information on NSAIDs exposure. The summary ORs were $0.82(95 \% \mathrm{CI}: 0.73$ - 0.92) pooled from six questionnaire-based studies $[11,23-25,31,33]$ and 
1.14 (95\%CI: 1.02 - 1.28) from four studies based on prescription [26, 28, 29, 32]. These results suggested a significant protective effect was observed only in studies based on questionnaire.

In subgroup analyses by varied adjustment factors including age, gender, smoking, alcohol drink and body mass index (BMI), the association between NSAIDs use and the risk of HNC were non-significant in all strata (Table 2).

\section{DISCUSSION}

The findings from this meta-analysis of eleven observational studies, including 370,000 participants and 10,673 HNC cases, did not indicate overall NSAIDs use was significantly associated with a decreased risk for HNC.

When stratified by type of drugs, there was no association between use of aspirin or NA-NSAIDs and the risk of HNC. However, we observed a significant risk reduction of $15 \%$ in $\mathrm{HNC}$ risk for users of ibuprofen alone $(\mathrm{OR}=0.85 ; 95 \% \mathrm{CI}, 0.72-0.99)$. It was suggested that different types of NSAIDs might have different effects due to biological mechanisms $[34,35]$. Andrews et al. had demonstrated that ibuprofen was more effective at reducing cancer cell growth and survival across a variety of cancer cell lines compared to other NSAIDs [36, 37], which may explain our findings.

It is widely accepted that any potential protective effects of NSAIDs use against cancers are likely to involve a considerable duration [38]. Previous large randomized trials and cohort studies showed long-term use of aspirin and other NSAIDs have almost consistently been associated with a stronger reduced risk of colorectal cancer [39-41]. The time-risk relations are similar to those described for colorectal cancer, our results also observed a significant association between long-term aspirin use and the risk of $\mathrm{HNC}(\mathrm{OR}=0.78 ; 95 \% \mathrm{CI}, 0.67-0.92)$.

A significant preventive effect of NSAIDs use on $\mathrm{HNC}$ risk was observed in questionnaire-based studies but not in prescription-based studies. Studies that use prescription databases have their own shortcomings. Generally, no data were available regarding the use of over-the-counter medications including aspirin and NSAIDs, which will have underestimated exposure to these drugs. However, results from questionnaire-based

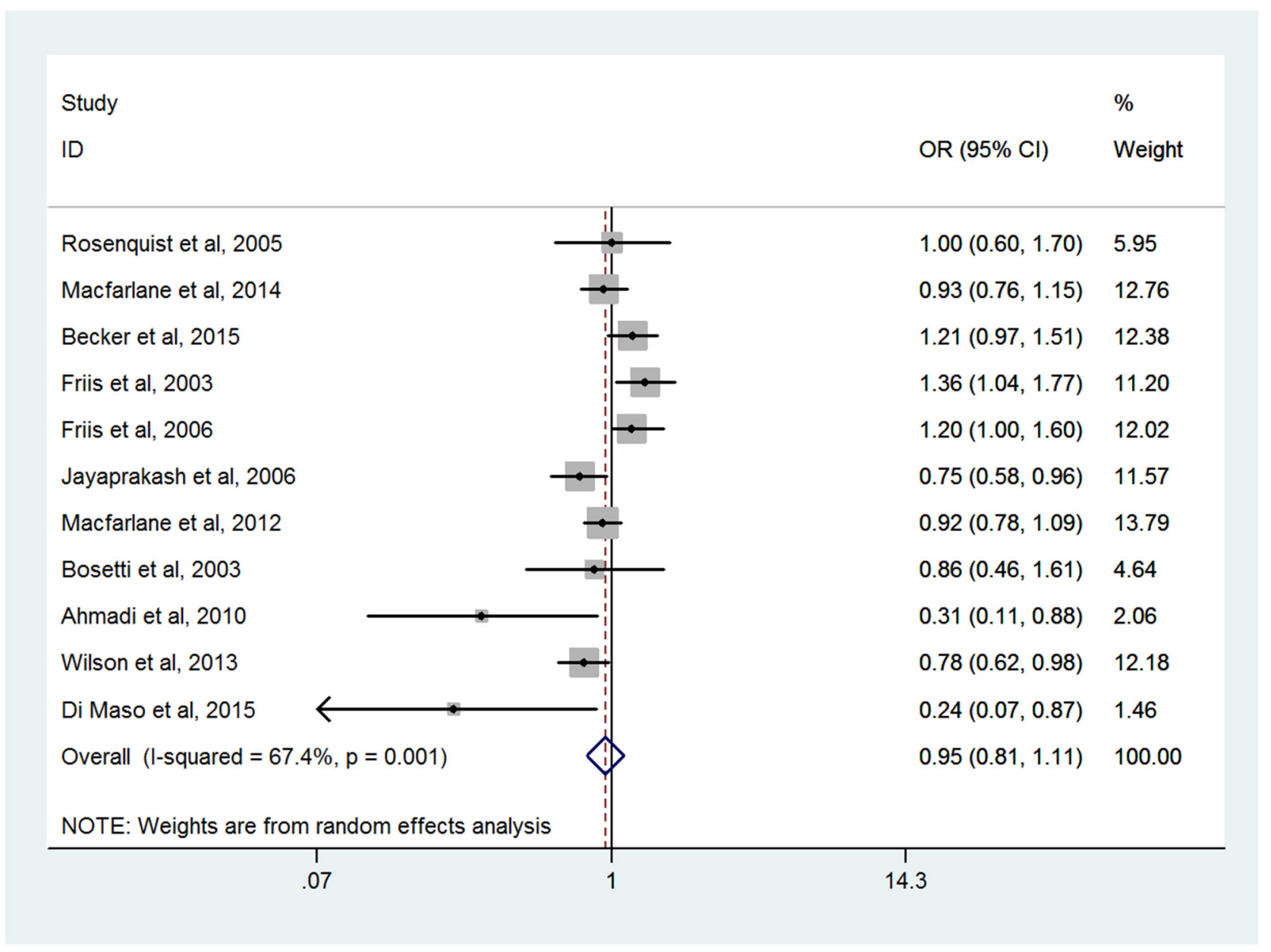

Figure 2: Forest plots of meta-analysis of overall NSAIDs use and the risk of HNC. 
Table 3: Associations between NSAIDs use and the risk of HNC in subgroup meta-analyses

\begin{tabular}{|c|c|c|c|c|c|c|c|}
\hline \multirow[t]{2}{*}{ Studies groups } & \multirow{2}{*}{$\begin{array}{l}\text { No. of } \\
\text { Studies }\end{array}$} & \multirow{2}{*}{$\begin{array}{c}\text { Summary OR } \\
(95 \% \text { CI })\end{array}$} & \multicolumn{2}{|c|}{ Homogeneity } & \multirow[t]{2}{*}{ Model used } & \multicolumn{2}{|c|}{ Publication Bias } \\
\hline & & & $P$-value & $I^{2}(\%)$ & & $\begin{array}{l}\text { Begg's } \\
P \text {-value }\end{array}$ & $\begin{array}{l}\text { Egger's } \\
P \text {-value }\end{array}$ \\
\hline \multicolumn{8}{|l|}{ Type of drugs use } \\
\hline Aspirin & 10 & $0.93(0.79-1.10)$ & 0.002 & $65.0 \%$ & Random-effects & 0.474 & 0.255 \\
\hline NA-NSAIDs & 4 & $0.92(0.76-1.10)$ & 0.026 & $67.7 \%$ & Random-effects & 0.734 & 0.342 \\
\hline Ibuprofen & 2 & $0.85(0.72-0.99)$ & 0.199 & $39.5 \%$ & Fixed-effects & 1.000 & NA \\
\hline $\begin{array}{l}\text { Long-term } \\
\text { aspirin use }\end{array}$ & 4 & $0.75(0.65-0.85)$ & 0.103 & $51.5 \%$ & Fixed-effects & 0.308 & 0.313 \\
\hline \multicolumn{8}{|l|}{ HNC sites } \\
\hline $\begin{array}{l}\text { Oral and } \\
\text { oropharynx }\end{array}$ & 5 & $1.01(0.85-1.20)$ & 0.098 & $43.9 \%$ & Random-effects & 0.230 & 0.139 \\
\hline Larynx & 3 & $0.96(0.65-1.42)$ & 0.059 & $64.7 \%$ & Random-effects & 0.296 & 0.253 \\
\hline Hypopharynx & 2 & $0.62(0.38-1.01)$ & 0.469 & $0.0 \%$ & Fixed-effects & 1.000 & NA \\
\hline Nasopharynx & 2 & $0.50(0.14-1.76)$ & 0.099 & $63.2 \%$ & Random-effects & 1.000 & NA \\
\hline \multicolumn{8}{|l|}{ Study design } \\
\hline Case-control & 8 & $0.89(0.74-1.07)$ & 0.017 & $59.1 \%$ & Random-effects & 0.108 & 0.130 \\
\hline Cohort & 3 & $1.08(0.77-1.51)$ & 0.003 & $82.4 \%$ & Random-effects & 0.296 & 0.446 \\
\hline \multicolumn{8}{|l|}{ Sample sizes } \\
\hline$\geqq 1000$ & 8 & $0.99(0.85-1.15)$ & 0.003 & $67.1 \%$ & Random-effects & 0.711 & 0.897 \\
\hline$<1000$ & 3 & $0.47(0.18-1.27)$ & 0.031 & $71.1 \%$ & Random-effects & 0.296 & 0.059 \\
\hline \multicolumn{8}{|l|}{ Exposure source } \\
\hline Questionnaire & 6 & $0.82(0.73-0.92)$ & 0.090 & $47.5 \%$ & Random-effects & 0.060 & 0.040 \\
\hline $\begin{array}{l}\text { Prescription } \\
\text { database }\end{array}$ & 4 & $1.14(1.02-1.28)$ & 0.118 & $48.9 \%$ & Fixed-effects & 0.308 & 0.134 \\
\hline \multicolumn{8}{|l|}{$\begin{array}{l}\text { Adjustments for } \\
\text { age }\end{array}$} \\
\hline Yes & 8 & $0.93(0.79-1.11)$ & 0.003 & $68.0 \%$ & Random-effects & 0.711 & 0.478 \\
\hline No & 3 & $0.89(0.51-1.53)$ & 0.039 & $69.3 \%$ & Random-effects & 0.296 & 0.233 \\
\hline \multicolumn{8}{|l|}{$\begin{array}{l}\text { Adjustments for } \\
\text { gender }\end{array}$} \\
\hline Yes & 8 & $0.93(0.79-1.11)$ & 0.003 & $68.0 \%$ & Random-effects & 0.711 & 0.478 \\
\hline No & 3 & $0.89(0.51-1.53)$ & 0.039 & $69.3 \%$ & Random-effects & 0.296 & 0.233 \\
\hline \multicolumn{8}{|l|}{$\begin{array}{l}\text { Adjustments for } \\
\text { smoking }\end{array}$} \\
\hline Yes & 7 & $0.88(0.73-1.06)$ & 0.022 & $59.6 \%$ & Random-effects & 0.230 & 0.382 \\
\hline No & 4 & $1.05(0.78-1.40)$ & 0.011 & $73.2 \%$ & Random-effects & 1.000 & 0.454 \\
\hline \multicolumn{8}{|l|}{$\begin{array}{l}\text { Adjustments for } \\
\text { alcohol }\end{array}$} \\
\hline Yes & 5 & $0.95(0.79-1.14)$ & 0.083 & $51.5 \%$ & Random-effects & 1.000 & 0.908 \\
\hline No & 6 & $0.91(0.69-1.21)$ & $<0.001$ & $77.5 \%$ & Random-effects & 1.000 & $\begin{array}{l}0.229 \\
(\text { Continued })\end{array}$ \\
\hline
\end{tabular}




\begin{tabular}{|c|c|c|c|c|c|c|c|}
\hline \multirow[t]{2}{*}{ Studies groups } & \multirow{2}{*}{$\begin{array}{l}\text { No. of } \\
\text { Studies }\end{array}$} & \multirow{2}{*}{$\begin{array}{c}\text { Summary OR } \\
(95 \% \mathrm{CI})\end{array}$} & \multicolumn{2}{|c|}{ Homogeneity } & \multirow[t]{2}{*}{ Model used } & \multicolumn{2}{|c|}{ Publication Bias } \\
\hline & & & $P$-value & $I^{2}(\%)$ & & $\begin{array}{l}\text { Begg's } \\
P \text {-value }\end{array}$ & $\begin{array}{l}\text { Egger's } \\
P \text {-value }\end{array}$ \\
\hline \multicolumn{8}{|l|}{$\begin{array}{l}\text { Adjustments for } \\
\text { BMI }\end{array}$} \\
\hline Yes & 3 & $0.95(0.76-1.20)$ & 0.023 & $73.6 \%$ & Random-effects & 1.000 & 0.939 \\
\hline No & 8 & $0.92(0.72-1.17)$ & 0.002 & $69.4 \%$ & Random-effects & 0.386 & 0.164 \\
\hline
\end{tabular}

Abbreviations: BMI, body mass index; CI, confidence interval; HNC, Head and Neck Cancers; NA, not available; NA-NSAIDs, non-aspirin nonsteroidal anti-inflammatory drugs; NSAIDs, nonsteroidal anti-inflammatory drugs; OR, odds ratio.

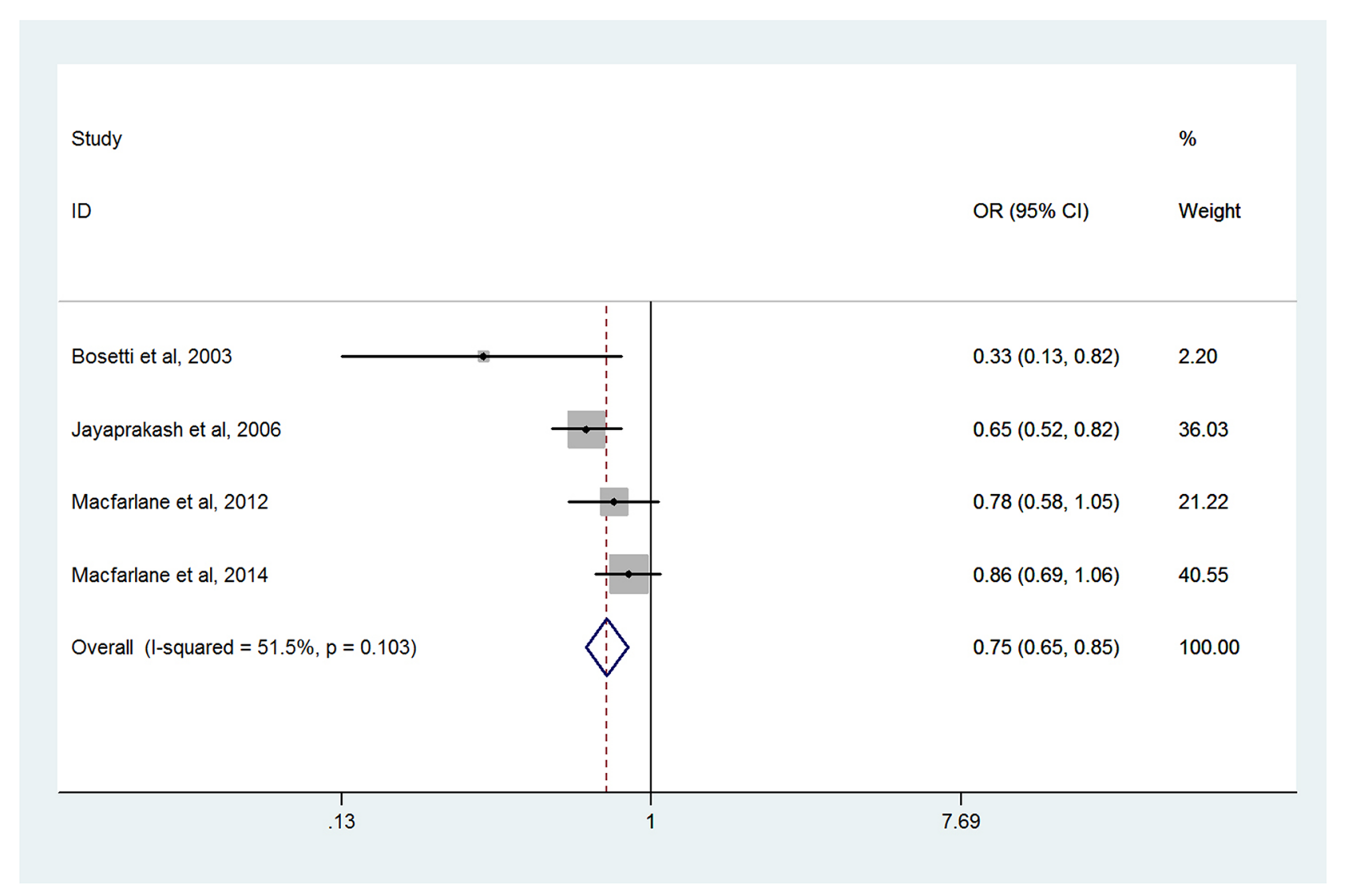

Figure 3: Forest plots of meta-analysis of long-term usage of aspirin and the risk of HNC.

studies should be interpreted cautiously for recall bias or selection bias. If the relatively healthy aspirin users were more likely to participate in the study than non-users, and an overestimate inverse association could be observed.

The previous systematic review including 2 cohort studies [28, 29] and 3 case-control studies [11, 23, 30], conducted by Wilson et al. also suggested no definitive conclusion can be reached on the preventive effect of NSAIDs on HNC risk [27]. The strengths of our metaanalysis were as follows: First, our present study included sufficient cases and quantitatively analyzed the effect of NSAIDs/aspirin using a detailed meta-analysis of eleven observational studies; Second, we were more capable to investigate potentially different effects on risk by the type and duration of NSAIDs use. As mentioned in the conclusion of Wilson's systematic review, aspirin may protect against HNC. We not only found a significant risk reduction in $\mathrm{HNC}$ risk for long-term aspirin but also ibuprofen user; Third, we performed the publication bias and more subgroup analyses, which reinforce our confidence in the validity of the conclusion.

There were some potential limitations that have to be considered when interpreting these results. First, this meta-analysis is based on observational studies, which are 
more susceptible to recall and selection biases, and could result in an underestimation or an overestimation of the true effect. However, It may be unfeasible to evaluate the long-term protective effects of NSAID from randomized clinical trials due to the large sample size required; Second, we did not take into account possible interactions with other drugs due to absence of data. Use of NSAIDs are often associated with other drugs use, which could have concealed a possible association with NSAIDs [42]. Third, none of the studies included adjusted the analyses for HPV infection, which has been shown to have an etiological role in HNC as well as smoking and heavy alcohol drink, and there was some evidence to suggest an up-regulation of COX-2 in HPV-infected tissues [43, 44]. Fourth, HNC are a heterogeneous group of neoplasms, and we conducted subgroup analyses separately for specific HNC sites, however no significant risk reduction was found; Fifth, the possibility of publication bias is always a concern in meta-analyses of published studies. This could bias the results of this review if negative studies were less likely to be published. In our meta-analysis, a significant publication bias in the subgroup of long-term aspirin use was observed from the Egger's test $(\mathrm{P}=0.055)$ but not the Begg's test $(\mathrm{P}=0.308)$. We consider the discrepancy was due to the small number of studies included.

In conclusion, our meta-analysis does not support the hypothesis that overall use of NSAIDs significant reduces the risk of $\mathrm{HNC}$. Whereas, we cannot rule out a modest reduction in $\mathrm{HNC}$ risk associated with ibuprofen and long-term aspirin use. Further large-scale robust studies are required, in particular, those evaluating the duration of aspirin use that may be take a protective effect.

\section{MATERIALS AND METHODS}

\section{Publication search}

This meta-analysis was conducted according to the meta-analysis of Observational Studies in Epidemiology (MOOSE) Guidelines [45]. We systematically searched Pubmed, Embase, Google scholar, and Cochrane library for manuscripts that mentioned the relationship between the use of aspirin and NSAIDs and the risk of HNC without language restriction, from January 1980 to April 2016. Our search terms consisted of three main components, head and neck (head and neck OR oral OR oropharynx OR hypopharynx OR larynx OR upper aerodigestive tract) AND disease (cancer OR neoplasms OR carcinoma) AND the exposure factor (aspirin OR NSAIDs OR ibuprofen OR naproxen OR indomethacin OR meloxicam OR valdecoxib OR celecoxib OR rofecoxib). We also reviewed the reference lists of articles with information on the topic to retrieve additional pertinent studies. If necessary, we attempted to contact the authors if we required additional information.

\section{Study selection}

Studies that met the following criteria were eligible for inclusion: (1) use a case-control or cohort study design; (2) evaluate the association between NSAIDs use and the risk of $\mathrm{HNC}$; (3) provided the odds ratio (OR) or relative risk (RR) with corresponding confidence interval (CI) or sufficient data to calculate them. When the same author reported results obtained from the same population in more than one publication, only the most recent report, or the most complete one, was included in the analysis. Data from review articles, case reports, abstracts, and letters were not included.

\section{Data extraction and quality assessment}

Two investigators (Lanhua Tang and Huabin $\mathrm{Hu}$ ) extracted the following information from each eligible studies independently: the last name of the first author, year of publication, study design, country where the study was performed, enrollment periods, HNC sites, sample size (numbers of case patients and control subjects), types of NSAIDs use, the source of NSAIDs exposure information, the source of HNC diagnosis, the ORs or RRs with corresponding 95\% CI and adjustment for covariates. Differences in data extraction were resolved by consensus, referring back to the original article.

The methodological quality of the included studies was assessed using the 9-star Newcastle-Ottawa scale for quality of nonrandomized studies in meta-analyses [46]. The Newcastle-Ottawa Scale consists of eight items, which are categorized three categories: selection (one star each), comparability (up to two stars), and exposure/ outcome (one star each). A "star" presents a "high-quality" choice of individual study.

\section{Statistical analysis}

Odds ratio (OR) was used as a measure of the association between use of NSAIDs and risk of HNC. Because the absolute risk of HNC is low, the OR in casecontrol studies was considered reasonable approximations of the corresponding rate ratios in cohort studies [47]. The heterogeneity of the estimators of OR was tested by Cochran's Q test at the $P<0.10$ level of significance [48]. We also calculated the quantity $I^{2}$ that describes the percentage variation across studies that is attributed to heterogeneity $[49,50]$. When significant heterogeneity was found, the random-effects model with the DerSimonianLaird method was used for meta-analysis [51]. Otherwise, the fixed-effects model with Mantel-Haenszel method was adopted [52]. Publication bias was evaluated using the Begg's adjusted rank correlation test [53], and the Egger's regression asymmetry test [54]. $P<0.10$ was considered to represent statistically significant publication bias.

When study reported results separately for aspirin and non-aspirin NSAIDs (NA-NSAIDs), to avoid double 
counting of the cases, we included only the results for aspirin in the overall summary estimate. Use of NSAIDs has the definition as follows:"overall use" was all the reported intake levels of NSAIDs use, "long-term use" was defined the duration of NSAIDs use or the time between last and first prescription more than 5 years. We only calculated the results for combined sites of HNC in the overall estimate, if studies had results for specific site and combined sites of HNC.

Analysis was performed using the STATA version 11.0 (Stata Corporation, College Station, Texas).

\section{ACKNOWLEDGMENTS AND GRANT SUPPORT}

This work was supported by grants from the National Natural Science Foundation of China (No: 81502658); the National Key Clinical Specialty, Oncology Department (National Health and Family Planning Commission of the PRC 2013/544).

\section{CONFLICTS OF INTEREST}

The authors declare no potential conflicts of interest.

\section{REFERENCES}

1. Ferlay J, Soerjomataram I, Dikshit R, Eser S, Mathers C, Rebelo M, Parkin DM, Forman D and Bray F. Cancer incidence and mortality worldwide: sources, methods and major patterns in GLOBOCAN 2012. International journal of cancer. 2015; 136:E359-386.

2. Hashibe M, Brennan P, Benhamou S, Castellsague X, Chen C, Curado MP, Dal Maso L, Daudt AW, Fabianova E, Fernandez L, Wunsch-Filho V, Franceschi S, Hayes RB, et al. Alcohol drinking in never users of tobacco, cigarette smoking in never drinkers, and the risk of head and neck cancer: pooled analysis in the International Head and Neck Cancer Epidemiology Consortium. Journal of the National Cancer Institute. 2007; 99:777-789.

3. Petti S. Lifestyle risk factors for oral cancer. Oral oncology. 2009; 45:340-350.

4. Lagiou P, Talamini R, Samoli E, Lagiou A, Ahrens W, Pohlabeln H, Benhamou S, Bouchardy C, Slamova A, Schejbalova M, Merletti F, Richiardi L, Kjaerheim K, et al. Diet and upper-aerodigestive tract cancer in Europe: the ARCAGE study. International journal of cancer. 2009; 124:2671-2676.

5. Negri E, Boffetta P, Berthiller J, Castellsague X, Curado MP, Dal Maso L, Daudt AW, Fabianova E, Fernandez L, WunschFilho V, Franceschi S, Hayes RB, Herrero R, et al. Family history of cancer: pooled analysis in the International Head and Neck Cancer Epidemiology Consortium. International journal of cancer. 2009; 124:394-401.
6. Dayyani F, Etzel CJ, Liu M, Ho CH, Lippman SM and Tsao AS. Meta-analysis of the impact of human papillomavirus (HPV) on cancer risk and overall survival in head and neck squamous cell carcinomas (HNSCC). Head \& neck oncology. 2010; 2:15.

7. Meurman JH. Infectious and dietary risk factors of oral cancer. Oral oncology. 2010; 46:411-413.

8. Chaturvedi AK, Engels EA, Anderson WF and Gillison ML. Incidence trends for human papillomavirusrelated and -unrelated oral squamous cell carcinomas in the United States. Journal of clinical oncology. 2008; 26:612-619.

9. Chang JS, Lo HI, Wong TY, Huang CC, Lee WT, Tsai ST, Chen KC, Yen CJ, Wu YH, Hsueh WT, Yang MW, Wu SY, Chang KY, et al. Investigating the association between oral hygiene and head and neck cancer. Oral oncology. 2013; 49:1010-1017.

10. Khuri FR, Kim ES, Lee JJ, Winn RJ, Benner SE, Lippman $\mathrm{SM}, \mathrm{Fu} \mathrm{KK}$, Cooper JS, Vokes EE, Chamberlain RM, Williams B, Pajak TF, Goepfert H, et al. The impact of smoking status, disease stage, and index tumor site on second primary tumor incidence and tumor recurrence in the head and neck retinoid chemoprevention trial. Cancer epidemiology, biomarkers \& prevention. 2001; 10:823-829.

11. Jayaprakash V, Rigual NR, Moysich KB, Loree TR, Nasca MA, Menezes RJ and Reid ME. Chemoprevention of head and neck cancer with aspirin: a case-control study. Archives of otolaryngology--head \& neck surgery. 2006; 132:1231-1236.

12. Tanaka T, Nishikawa A, Mori Y, Morishita Y and Mori H. Inhibitory effects of non-steroidal anti-inflammatory drugs, piroxicam and indomethacin on 4-nitroquinoline 1-oxideinduced tongue carcinogenesis in male $\mathrm{ACI} / \mathrm{N}$ rats. Cancer letters. 1989; 48:177-182.

13. Scioscia KA, Snyderman CH, D'Amico F, Comsa S, Rueger $\mathrm{R}$ and Light $\mathrm{B}$. Effects of arachidonic acid metabolites in a murine model of squamous cell carcinoma. Head \& neck. 2000; 22:149-155.

14. Lin DT, Subbaramaiah K, Shah JP, Dannenberg AJ and Boyle JO. Cyclooxygenase-2: a novel molecular target for the prevention and treatment of head and neck cancer. Head \& neck. 2002; 24:792-799.

15. Ulrich CM, Bigler $\mathrm{J}$ and Potter JD. Non-steroidal antiinflammatory drugs for cancer prevention: promise, perils and pharmacogenetics. Nature reviews Cancer. 2006; 6:130-140.

16. Thun MJ. Beyond willow bark: aspirin in the prevention of chronic disease. Epidemiology. 2000; 11:371-374.

17. Thun MJ, Henley SJ and Patrono C. Nonsteroidal antiinflammatory drugs as anticancer agents: mechanistic, pharmacologic, and clinical issues. Journal of the National Cancer Institute. 2002; 94:252-266. 
18. Sabichi AL and Lippman SM. COX-2 inhibitors and other nonsteroidal anti-inflammatory drugs in genitourinary cancer. Seminars in oncology. 2004; 31:36-44.

19. Pentland AP, Schoggins JW, Scott GA, Khan KN and Han R. Reduction of UV-induced skin tumors in hairless mice by selective COX-2 inhibition. Carcinogenesis. 1999; 20:1939-1944.

20. Mahmud SM, Franco EL and Aprikian AG. Use of nonsteroidal anti-inflammatory drugs and prostate cancer risk: a meta-analysis. International journal of cancer. 2010; 127:1680-1691.

21. Luo T, Yan HM, He P, Luo Y, Yang YF and Zheng H. Aspirin use and breast cancer risk: a meta-analysis. Breast cancer research and treatment. 2012; 131:581-587.

22. Wang WH, Huang JQ, Zheng GF, Lam SK, Karlberg J and Wong BC. Non-steroidal anti-inflammatory drug use and the risk of gastric cancer: a systematic review and metaanalysis. Journal of the National Cancer Institute. 2003; 95:1784-1791.

23. Bosetti C, Talamini R, Franceschi S, Negri E, Garavello $\mathrm{W}$ and La Vecchia C. Aspirin use and cancers of the upper aerodigestive tract. British journal of cancer. 2003; 88:672-674.

24. Wilson JC, Murray LJ, Hughes CM, Black A and Anderson LA. Non-steroidal anti-inflammatory drug and aspirin use and the risk of head and neck cancer. British journal of cancer. 2013; 108:1178-1181.

25. Macfarlane TV, Macfarlane GJ, Thakker NS, Benhamou S, Bouchardy C, Ahrens W, Pohlabeln H, Lagiou P, Lagiou A, Castellsague X, Agudo A, Slamova A, Plzak $\mathrm{J}$, et al. Role of medical history and medication use in the aetiology of upper aerodigestive tract cancers in Europe: the ARCAGE study. Annals of oncology. 2012; 23:1053-1060.

26. Macfarlane TV, Lefevre $\mathrm{K}$ and Watson MC. Aspirin and non-steroidal anti-inflammatory drug use and the risk of upper aerodigestive tract cancer. British journal of cancer. 2014; 111:1852-1859.

27. Wilson JC, Anderson LA, Murray LJ and Hughes CM. Nonsteroidal anti-inflammatory drug and aspirin use and the risk of head and neck cancer: a systematic review. Cancer causes \& control. 2011; 22:803-810.

28. Friis S, Sorensen HT, McLaughlin JK, Johnsen SP, Blot WJ and Olsen JH. A population-based cohort study of the risk of colorectal and other cancers among users of low-dose aspirin. British journal of cancer. 2003; 88:684-688.

29. Friis S, Poulsen A, Pedersen L, Baron JA and Sorensen HT. Use of nonsteroidal anti-inflammatory drugs and risk of oral cancer: a cohort study. British journal of cancer. 2006; 95:363-365.

30. Rosenquist K, Wennerberg J, Schildt EB, Bladstrom A, Goran Hansson B and Andersson G. Oral status, oral infections and some lifestyle factors as risk factors for oral and oropharyngeal squamous cell carcinoma. A population-based case-control study in southern Sweden. Acta oto-laryngologica. 2005; 125:1327-1336.

31. Ahmadi N, Goldman R, Seillier-Moiseiwitsch F, Noone AM, Kosti $\mathrm{O}$ and Davidson BJ. Decreased risk of squamous cell carcinoma of the head and neck in users of nonsteroidal anti-inflammatory drugs. International journal of otolaryngology. 2010; 2010:424161.

32. Becker C, Wilson JC, Jick SS and Meier CR. Non-steroidal anti-inflammatory drugs and the risk of head and neck cancer: A case-control analysis. International journal of cancer. 2015; 137:2424-2431.

33. Di Maso M, Bosetti C, La Vecchia C, Garavello W, Montella M, Libra M, Serraino D and Polesel J. Regular aspirin use and nasopharyngeal cancer risk: A case-control study in Italy. Cancer epidemiology. 2015; 39:545-547.

34. Hernandez-Diaz S and Garcia Rodriguez LA. Nonsteroidal anti-inflammatory drugs and risk of lung cancer. International journal of cancer. 2007; 120:1565-1572.

35. Vinogradova Y, Coupland C and Hippisley-Cox J. Exposure to cyclooxygenase-2 inhibitors and risk of cancer: nested casecontrol studies. British journal of cancer. 2011; 105:452-459.

36. Andrews P, Zhao X, Allen J, Li F and Chang M. A comparison of the effectiveness of selected non-steroidal anti-inflammatory drugs and their derivatives against cancer cells in vitro. Cancer chemotherapy and pharmacology. 2008; 61:203-214.

37. Andrews J, Djakiew D, Krygier S and Andrews P. Superior effectiveness of ibuprofen compared with other NSAIDs for reducing the survival of human prostate cancer cells. Cancer chemotherapy and pharmacology. 2002; 50:277-284.

38. Olsen JH. Interpretation in drug epidemiology. Lancet. 1998; 352:162-163.

39. Chan AT, Giovannucci EL, Meyerhardt JA, Schernhammer ES, Curhan GC and Fuchs CS. Long-term use of aspirin and nonsteroidal anti-inflammatory drugs and risk of colorectal cancer. JAMA. 2005; 294:914-923.

40. Flossmann E, Rothwell PM, British Doctors Aspirin T and the UKTIAAT. Effect of aspirin on long-term risk of colorectal cancer: consistent evidence from randomised and observational studies. Lancet. 2007; 369:1603-1613.

41. Rothwell PM, Wilson M, Elwin CE, Norrving B, Algra A, Warlow CP and Meade TW. Long-term effect of aspirin on colorectal cancer incidence and mortality: 20-year follow-up of five randomised trials. Lancet. 2010; 376:1741-1750.

42. Takkouche B, Etminan M, Caamano F and Rochon PA. Interaction between aspirin and ACE Inhibitors: resolving discrepancies using a meta-analysis. Drug safety. 2002; 25:373-378.

43. Zaravinos A. An updated overview of HPV-associated head and neck carcinomas. Oncotarget. 2014; 5:3956-3969. doi: 10.18632/oncotarget.1934.

44. Subbaramaiah K and Dannenberg AJ. Cyclooxygenase-2 transcription is regulated by human papillomavirus 16 E6 
and E7 oncoproteins: evidence of a corepressor/coactivator exchange. Cancer research. 2007; 67:3976-3985.

45. Stroup DF, Berlin JA, Morton SC, Olkin I, Williamson GD, Rennie D, Moher D, Becker BJ, Sipe TA and Thacker SB. Meta-analysis of observational studies in epidemiology: a proposal for reporting. Meta-analysis Of Observational Studies in Epidemiology (MOOSE) group. JAMA. 2000; 283:2008-2012.

46. Wells GA, Shea B, O'Connell D, Peterson J, Welch V, Losos M and Tugwell P. (2010). The Newcastle-Ottawa Scale (NOS) for assessing the quality of nonrandomised studies in meta-analyses. http:/www.ohri.ca/programs/ clinical_epidemiology/oxford.asp.

47. Zhang J and Yu KF. What's the relative risk? A method of correcting the odds ratio in cohort studies of common outcomes. JAMA. 1998; 280:1690-1691.

48. Cochran WG. The Combination of Estimates from Different Experiments. Biometrics. 1954; 10:101-129.
49. Higgins JP and Thompson SG. Quantifying heterogeneity in a meta-analysis. Statistics in medicine. 2002; 21:1539-1558.

50. Higgins JP, Thompson SG, Deeks JJ and Altman DG. Measuring inconsistency in meta-analyses. BMJ. 2003; 327:557-560.

51. DerSimonian R and Laird N. Meta-analysis in clinical trials. Controlled clinical trials. 1986; 7:177-188.

52. Mantel N and Haenszel W. Statistical aspects of the analysis of data from retrospective studies of disease. Journal of the National Cancer Institute. 1959; 22:719-748.

53. Begg CB and Mazumdar M. Operating characteristics of a rank correlation test for publication bias. Biometrics. 1994; 50:1088-1101.

54. Egger M, Davey Smith G, Schneider M and Minder C. Bias in meta-analysis detected by a simple, graphical test. BMJ. 1997; 315:629-634. 